\title{
Enseñar historia: el desafío de formar estudiantes críticos y reflexivos
}

\author{
Teach history: the challenge of forming critical and \\ reflective students
}

\section{Ensinar história: o desafio de formar alunos críticos e reflexivos}

\author{
María Gabriela Pauli \\ Doctora en educación y doctora en historia \\ Universidad Católica de Santa Fe de Argentina \\ gabrielapaulidegarcia@gmail.com
}

\section{Resumen}

Escribir sobre la enseñanza de la Historia es siempre problematizar. Problematizar por un lado, porque la tarea educativa es una práctica sumamente compleja y no caben entonces las miradas lineales; problematizar porque la Historia, en tanto se ocupa de los hombres, de sus acciones, de sus decisiones, de las estructuras sociales, económicas, políticas y culturales que ellos han gestado en el tiempo, es también una disciplina de una densidad y complejidad muy notorias.

La pretensión del presente artículo es reflexionar en torno a las posibilidades de enseñar Historia para favorecer el pensamiento crítico y reflexivo de nuestros estudiantes de la escuela secundaria. Lo haremos situándonos en la educación argentina por dos razones: en primer lugar, no es posible escribir desde ningún lugar, como historiadores, sabemos que todo proceso es situado y éste no escapa a esa condición. En segundo lugar, porque es la realidad que conocemos, y sobre la que podemos reflexionar. El deseo es que estas líneas sirvan para ayudar a pensar la enseñanza de la Historia en otros escenarios, con las particularidades de cada uno de ellos en función de ciertos elementos comunes que posibilitan que una experiencia situada pueda compartirse.

Palabras clave: Historia de la educación, enseñanza de la historia, educación en Argentina 


\begin{abstract}
Writing about the teaching of History is always problematizing. Problematize on the one hand, because the educational task is an extremely complex practice and linear views do not fit; problematize because History, while it deals with men, their actions, their decisions, the social, economic, political and cultural structures that they have developed over time, is also a discipline of a very well-known density and complexity.

The aim of this article is to reflect on the possibilities of teaching History to favor the critical and reflective thinking of our high school students. We will do so by placing ourselves in Argentine education for two reasons: first, it is not possible to write from anywhere, as historians, we know that all processes are located and this does not escape that condition. Second, because it is the reality we know, and on which we can reflect. The desire is that these lines serve to help think the teaching of History in other scenarios, with the particularities of each one of them based on certain common elements that enable a situated experience to be shared.
\end{abstract}

Keywords: History of education, teaching of history, education in Argentina

\title{
Resumo
}

Escrever sobre o ensino da História é sempre problematizador. Problematize, por um lado, porque a tarefa educacional é uma prática extremamente complexa e as visões lineares não se encaixam; problematizar porque a História, enquanto trata dos homens, suas ações, suas decisões, as estruturas sociais, econômicas, políticas e culturais que desenvolveram ao longo do tempo, é também uma disciplina de densidade e complexidade muito conhecidas.

O objetivo deste artigo é refletir sobre as possibilidades do ensino da História para favorecer o pensamento crítico e reflexivo de nossos alunos do ensino médio. Faremos isso inserindo-nos na educação argentina por dois motivos: primeiro, não é possível escrever de qualquer lugar, como historiadores, sabemos que todos os processos estão localizados e isso não foge a essa condição. Segundo, porque é a realidade que conhecemos e sobre a qual podemos refletir. O desejo é que essas linhas sirvam para ajudar a pensar o ensino da História em outros cenários, com as particularidades de cada uma delas baseadas em certos elementos comuns que possibilitam uma experiência situada a ser compartilhada.

Palavras-chave: História da educação, ensino de história, educação na Argentina 
Una apretada síntesis del marco de esta reflexión que proponemos

El proceso de transformación educativa en Argentina que se inició en la década de los 90 dio lugar a una nutrida producción de trabajos vinculados a la enseñanza de la Historia, desde perspectivas didácticas o psicológicas, e incluso combinando ambas. Muchos de ellos se abocaron a desarrollar propuestas didácticas para una enseñanza renovada de la Historia, exponiendo estrategias novedosas así como formulaciones alternativas de contenidos y de los modos de presentarlos. Además de la producción local, la escuela secundaria argentina abrevó en artículos producidos en el contexto de implementación de la LOGSE ${ }^{1}$ en España, como fueron los de Mario Carretero y su equipo, los de Joaquim Prats, Joan Pagés o Cristofol Trepat, por mencionar los autores que más circularon entre los docentes.

A su vez, los profesores de las universidades nacionales argentinas vinculados a las cátedras de Didáctica de la Historia y a otros espacios curriculares afines, han constituido una asociación - APEHUN - y con regular frecuencia organizan eventos académicos destinados a discutir los desarrollos y las problemáticas más recientes en el campo de la enseñanza de la Historia. También han generado publicaciones periódicas específicas que dan cuenta de la producción de conocimiento en relación a estos temas.

En todos los casos, el interés ha sido y sigue siendo el de evitar un aprendizaje memorístico y repetitivo de la Historia, favorecer la comprensión del pasado, y propiciar una actitud crítica ante el saber histórico.

Ante este panorama se impone la pregunta acerca de ¿qué podemos aportar a la reflexión pedagógica en torno a la enseñanza de la Historia, si se trata de un campo que cuenta con tan prolífica producción? ¿Es posible decir algo nuevo en torno al asunto?

Consideramos que es posible una mirada que pueda resultar un aporte para seguir pensando en el para qué de la enseñanza de la Historia en la escuela secundaria, partiendo desde la esencia misma de la a educación: la formación integral de los sujetos.

\section{Pensando el para qué enseñar Historia en clave antropológica y epistemológica}

Un primer ejercicio para pensar la enseñanza de la Historia será desprendernos de las explicaciones y propuestas de cambio simples. No se trata de cambiar tan sólo paradigmas historiográficos, diseños curriculares, estrategias

1 "Ley Orgánica del Sistema Educativo, que rigió entre los años 1990 y 200 
metodológicas o implementar el uso de otros recursos, si bien todo ello puede ser necesario. La cuestión radica en revisar nuestras concepciones más profundas en orden a los sujetos de la educación -el sujeto que aprende, pero también el que enseña, el que conduce la institución, el que limpia, y todos los sujetos que participan en alguna medida de la educación formal-. También habremos de revisar nuestra concepción epistémica en relación a la Historia.

Del modo en el que pensemos estas dos dimensiones, dependerá la posibilidad de enseñar Historia para favorecer el pensamiento crítico y reflexivo. En este punto conviene hacer una aclaración: no es posible pensar la enseñanza sin un encuadre filosóficoantropológico y sin un marco epistemológico. Toda propuesta pedagógica o didáctica que carece de estos encuadres explícitos, los asume de manera implícita. Acordamos con Jürgen Habermas (1990) en la necesidad de hacer explícitos estos supuestos desde los que se formulan las propuestas $^{2}$ para permitir una adecuada comprensión de las mismas, y también por honestidad intelectual.

Para pensar acerca de las posibilidades de una enseñanza de la Historia que contribuya a formar el pensamiento crítico y reflexivo

2 Ver sobre esta cuestión los aportes de Jürgen HABERMAS en relación a la acción comunicativa y sus presupuestos, en La lógica de las ciencias sociales. Tecnos, Madrid, 1990. de los estudiantes, nos posicionamos desde una antropología personalista y una epistemología de la Historia como el ámbito en el que se pone en juego la libertad de los seres humanos.

\section{La antropología personalista y sus posibilidades para pensar a los sujetos de la educación}

Varias razones nos han decidido a optar por una antropología de corte personalista. La de mayor peso, es la integralidad que caracteriza a la concepción del hombre ${ }^{3}$ en tanto persona y que nos permite abordar una multiplicidad de dimensiones, por otra parte, es la noción que asume el sistema educativo argentino en su legislación desde 1993.

Como es sabido, la conceptualización de la persona humana no es unívoca. Hay múltiples matices que se acentúan o suavizan según los autores. Resulta imposible en pocas páginas dar cuenta tan vasto universo, pero sí podemos proponer algunos rasgos que ubiquen al lector acerca de nuestro posicionamiento antropológico.

La vida humana implica la búsqueda de sentido, de un para qué vivir, una dirección que moviliza la existencia y constituye una permanente búsqueda. La

3 Usamos de manera genérica el término hombre como sinónimo de ser humano, para hacer más fácil la lectura. 
persona humana busca y da sentido a su vida, y a la vez dota de sentido a las cosas. Esta dimensión tiene una importancia pedagógica mayúscula, en la medida en que un sujeto en permanente búsqueda de sentido es alguien que se pregunta, que inquiere, que nunca cesa de indagar.

Las facultades específicamente humanas como son el pensamiento - capacidad de comprender el mundo y de saberse en él - y la libertad - capacidad de elegir el bien o al menos aquello que se presenta bajo apariencia de bien son distintivas del actuar humano y establecen la preeminencia del espíritu y el debilitamiento de lo instintivo, resultan una dimensión vastísima de lo educable y tienen una importante repercusión social.

El ser humano es un ser con otros, es en la relación Yo-Tú que la persona desarrolla sus dimensiones específicamente humanas tal como lo propone Martin Buber (1994) $)^{4}$ En el plano de la relación con los otros seres humanos, se instala el lenguaje, capacidad específicamente humana, en tanto lenguaje articulado y complejo, capaz de expresar emociones, sentimientos e ideas abstractas, a la vez que condición indispensable para la apertura al otro ${ }^{5}$.

\footnotetext{
${ }^{4}$ Ver el trabajo de Martin BUBER. Yo y tú. Ed. Nueva Visión, Bs As, 1994.

${ }^{5}$ No sólo nos referimos al lenguaje verbal, los gestos $y$ poses son formas de lenguaje también, la música, la matemática han generado sus propios lenguajes, todos ellos válidos en diferentes instancias.
}

La persona humana es un ser inacabado, abierto, que ha de completarse en la relación con otras personas y en el ámbito de lo sociocultural. Romano Guardini $(1967)^{6}$ afirma que la persona depende de que otras personas existan; sólo posee sentido la propia existencia, cuando hay otras personas con las que puede tener lugar el encuentro.

Es también un ser en el mundo, un ser situado en un tiempo y espacio concreto. El ejercicio de su libertad se inserta en la dimensión histórico-cultural de la existencia humana. Libertad que es condición y tarea, en la medida en que se crece en el ejercicio de esta facultad de modo de poder ser más libre cuanto más consciente se es de las decisiones a tomar y sus implicancias.

La concepción personalista reconoce en el hombre una dimensión trascendente, que define de este modo el plano de la interioridad y la posesión de sí ${ }^{7}, y$ una dimensión relacional -no sólo con respecto a otros seres humanos sino a la trascendencia-, ambas constitutivas del ser persona.

Todas estas dimensiones del ser humano pensado como persona, implican una producción

\footnotetext{
${ }^{6}$ Guardini, Romano. Mundo y Persona. Ensayos para una teoría cristiana del hombre. $1^{\mathrm{a}}$ ed. en castellano 1963. Ed. Guadarrama, Madrid, 1967.

7 Dirá Guardini que la persona humana es un misterio insondable aún para sí misma. Ver Mundo y persona....
} 
específicamente humana como es la cultura. Lo propiamente humano es la cultura: podríamos afirmar que ella misma es la naturaleza del ser humano. En todo acto de cultura hay una apropiación personal, creativa e irrepetible de la realidad circundante que implica la elaboración de respuestas a problemáticas personales o sociales, que son fruto de la inteligencia y del potencial transformador de los seres humanos. Todo acto de cultura se sustenta en una acción comunitaria: pensamos el mundo, recreamos la realidad, formulamos respuestas, proyectos y alternativas, en el marco de una serie de supuestos, esquemas valorativos, necesidades que nos vienen dados por otros. La creación de cultura nunca es una actividad individual, es siempre una tarea comunitaria y que no excluye el aporte personal de cada sujeto.

\section{La historia como ámbito en el que se pone en juego la libertad de los seres humanos y sus implicancias pedagógicas.}

El planteo antropológico que hemos reseñado, nos permite pensar la Historia no como algo fatídicamente dado, sino como una permanente construcción de las personas - individual y comunitaria o colectiva - de modo tal que todo lo que hacemos o dejamos de hacer, es Historia e influye en el curso de los acontecimientos.
El hombre - los seres humanos hacen Historia y a la vez, se van conformando ellos mismos en esa historia que transitan y transforman. Esto presupone una responsabilidad social $y$ exige asumir el protagonismo personal irreemplazable de cada ser humano en la Historia, en su propia historia. Entonces, el modo en el que pensamos al hombre condiciona nuestra perspectiva epistemológica, no sólo sobre la construcción del conocimiento histórico, sino sobre la misma esencia de lo histórico.

¿Cómo pensamos la Historia desde un marco antropológico personalista, como el que explicitamos antes? Definimos a la Historia como el ámbito donde se pone en juego la libertad de la persona humana. La Historia, se construye así, a partir de las decisiones que cada hombre asume - y de las que no asume - a lo largo de su vida, decisiones que involucran siempre a otros tanto porque los afectan como porque esas decisiones están condicionadas por el entorno cultural. Una perspectiva cultural en relación al conocimiento de la Historia, nos posibilita reconocer en toda manifestación humana - y por lo tanto en toda manifestación histórica - una expresión de cultura, y asumir a la vez el ámbito de las decisiones personales y la dimensión de lo social comunitario.

Son protagonistas de la Historia todos los hombres y cada uno de ellos, en la medida en que cada 
persona es responsable de su proyecto personal de vida $y$ también de posibilitar las condiciones para que los demás hombres puedan realizar sus propios proyectos de vida. La Historia resulta entonces, una realidad sumamente compleja, impredecible, sujeta al cambio y donde se entrecruzan innumerables intencionalidades en un entramado muy denso. Esta característica torna a su vez apasionante el estudio de la Historia, ya que nunca son obvias las explicaciones, ni son unívocos los modos de pensar el pasado.

De la complejidad de lo humano deviene la complejidad de lo histórico, y ello obliga a pensar en estrategias metodológicas también complejas para abordar el conocimiento histórico. La historia - problema, tal como la proponen algunos historiadores de la Escuela de los Annales y de la Escuela Marxista británica, resulta adecuada a nuestras necesidades metodológicas; siempre que al asumir el carácter personal de toda empresa humana renunciemos a la posibilidad de lograr generalizaciones y regularidades como fin de nuestra investigación ${ }^{8}$; y admitamos que nuestra tarea de historiadores se propone comprender a los hombres: a cada hombre concreto que ha protagonizado su propia historia y alguna porción de la historia de la humanidad. A su vez, la Historia es

8 Podemos adjudicarle espacio a las conceptualizaciones teóricas y a la obtención de regularidades como medios para la comprensión de lo histórico; pero admitiendo hermenéutica, han dicho los historiadores de la Escuela Erudita alemana del siglo XIX, y esta condición, constituye también un criterio metodológico para la enseñanza de una Historia que favorezca el pensamiento crítico y reflexivo de los estudiantes de la escuela secundaria, en orden a formarlos como ciudadanos comprometidos con los derechos humanos y con la construcción de una nación justa y solidaria en la que haya oportunidades para todos.

La complejidad propia de la Historia, lejos de constituir una limitación, es su mayor riqueza. Ella nos posibilita abordar la complejidad de la vida humana individual y comunitaria - en toda su extensión y en toda su profundidad. Esto es así, en la medida en que la persona humana es un ser histórico, y por ello mismo todo lo humano es histórico, y contingente. El ser humano es histórico en cuanto su ser se desenvuelve en el tiempo. Por ello sus acciones, su pensamiento - y esto incluye la ciencia, las manifestaciones artísticas, las concepciones políticas, los modelos económicos, las formas de organización social son productos históricamente conformados y por ello mismo contingentes. La multiplicidad de métodos y la pluralidad de enfoques, resultan así útiles en la medida en que nos proporcionen

que la Historia siempre trasciende la esquematización propia de estos niveles de pensamiento. 
algunos elementos para ese acercamiento.

Por ello, no sólo resulta importante aprender Historia, sino que es necesario. Y lo es para todos los estudiantes de la educación secundaria, en la medida en que, el conocimiento histórico posibilita situar otros aprendizajes y de este modo contribuye a dotarlos de sentido. Así, por ejemplo, la historia de la ciencia permite ver como se gestan los conocimientos científicos, para comprender que no surgen espontáneamente y que no son ajenos a la vida, a las preocupaciones y a las necesidades de los hombres y de los pueblos en que esas ciencias han surgido y se han desarrollado. Lo mismo ocurre en relación al conocimiento filosófico: la Historia nos posibilita apreciar como las ideas, las teorías y los sistemas filosóficos han sido respuesta a las inquietudes humanas de determinados pueblos y sociedades, y a las necesidades de los hombres de darse respuesta a sus interrogantes más hondos y así podríamos continuar ejemplificando en relación a otras manifestaciones humanas como el arte, la literatura y la evolución del lenguaje, las creencias, etc.

Más aún, el conocimiento histórico nos muestra cómo, esos procesos que devienen en nuevos aportes al campo de la ciencia, del conocimiento filosófico $\mathrm{o}$ de cualquier otro ámbito de la realidad humana, son procesos mucho más complejos, y que esos aportes están siempre acompañados de cambios en la vida política y las instituciones, en la organización económica y social de los pueblos, en los valores, costumbres y sistemas de creencias. El conocimiento de los períodos de la historia de la humanidad y de las regularidades que permiten caracterizar a cada uno de ellos, constituyen herramientas metodológicas que posibilitan adentrarnos en las particularidades de cada acontecimiento y de cada proceso histórico.

Deriva de las afirmaciones precedentes, la necesidad de enseñar a los adolescentes a pensar históricamente, Ahora bien, ello sólo es posible para quien conoce y comprende la lógica de la construcción del conocimiento histórico y puede desnaturalizar interpretaciones, hechos o procesos, asumiendo su carácter histórico y contingente.

Este ejercicio de pensar históricamente proporciona por un lado la posibilidad, de contextualizar los procesos en los que intervienen los seres humanos, y por otro lado, permite, desde un enfoque hermenéutico; la comprensión de lo más profundamente humano, en la medida en que los sitúa frente al hombre, frente a su libertad e inteligencia, frente a su posibilidad de ser con otros, frente a sus sentimientos y expectativas, frente a su generosidad y entrega, frente a sus temores y prejuicios, etc. En síntesis, nos sitúa frente a lo humano, con sus miserias y grandezas, con sus aciertos y sus 
errores, y en última instancia los enfrenta a las decisiones personales y a sus consecuencias que siempre resultan ser sociales.

De esta manera, el conocimiento y la comprensión de los procesos históricos, hacen posible desnaturalizar ${ }^{9}$ formas culturales y sociales, ideologías, teorías, criterios de análisis que resultan de procesos históricos - algunos de larga o muy larga duración- de la humanidad o de una porción significativa de ella, en tanto descubrimos su génesis en la Historia, las condiciones que han posibilitado su desarrollo y advertimos su carácter contingente. Es, en este sentido que enseñar Historia favorece el desarrollo del pensamiento crítico y reflexivo.

\section{Las condiciones para un pensamiento crítico y reflexivo}

Existe un interés renovado en los trabajos vinculados a la enseñanza de la Historia, en proponer estrategias que ayuden a la reflexión; y que formen sujetos

\footnotetext{
9 Tomamos el concepto de desnaturalización en el sentido en el que lo aborda y define Enrique Bambozzi: "El concepto problematizar lo interpretamos como sinónimo de desnaturalizar, no en el sentido de ir en contra de la naturaleza, sino de cuestionar aquello que siempre ha sido así, de cuestionar lo obvio, lo instituido, para desmontar su arqueología y capitalizar desde una posición críticopropositiva aquellos aspectos que nos permiten avanzar en construcciones cada vez más humanizadoras." Escritos pedagógicos. Ediciones del Copista, Córdoba, 2005. p. 13.

10 "Pero yo tomé deliberadamente la decisión de escribir acerca de las inteligencias múltiplemúltiples"e para resaltar el número
}

críticos. Ahora bien, entendemos que es posible darle más profundidad a este concepto y asumirlo como un pensamiento liberador que no puede sino ser crítico y reflexivo, en la medida en que le posibilite a la persona humana pensarse y pensar la realidad, y más aún pensarse en el mundo. Esto a su vez implica para cada uno de nosotros poder pensar $y$ hacer consciente las propias categorías desde las cuales estructuramos las ideas acerca de la realidad.

Hemos conjugado aportes diversos para formular una conceptualización en torno al pensamiento crítico y reflexivo que supere otras perspectivas en torno al asunto. Entre ellos, el concepto de inteligencias múltiples de Howard Gardner, por oposición a la consideración singular de la inteligencia, como única y homogénea. ${ }^{10}$ Gardner identifica siete inteligencias que son: la inteligencia lingüística y la lógicomatemática, que son aquellas que la sociedad moderna ${ }^{11}$ considera más importantes; la inteligencia

desconocido de capacidades humanas, desde la inteligencia musical hasta la inteligencia implicada en el conocimiento de uno mismo: e „inteligencias" para subrayar que estas capacidades son tan fundamentales como las que tradicionalmente detecta el test de CI." Gardner, Howard. Inteligencias múltiples. La teoría en la práctica. Paidós, Bs. As., 1998. p. 13.

11 En el sentido de Modernidad, es decir, la sociedad cuya cosmovisión se configura a partir del Renacimiento y alcanza su punto culminante con la Ilustración del siglo XVIII. 
espacial, la musical, la corporal y cinética, la inteligencia interpersonal y la intrapersonal. Como puede verse, a partir de la enumeración de las inteligencias, éstas abarcan las funciones que tradicionalmente se asocian al intelecto $y$ al pensamiento abstracto, pero también otras ligadas al dominio del cuerpo y de la relación con el espacio, al ámbito del arte y la creación de belleza, y al espacio de las relaciones personales y del autoconocimiento interior. Este planteo posibilita ampliar la dimensión del concepto de inteligencia, y nos ayuda a matizar la concepción acerca del pensamiento, actividad ligada indudablemente a la inteligencia.

También es insoslayable para formular nuestro concepto, el desarrollo teórico de Edgar Morin (2002), que explicita el paradigma de la complejidad acentuando el carácter de entramado de la realidad, y la necesidad para el abordaje de la misma, de pensar también en términos de complejidad $^{12}$. Este concepto acerca del pensamiento resulta más rico y de mayor amplitud; y sin embargo, constituye aún un concepto estrecho, que reduce el pensamiento al plano de las capacidades intelectuales, y que sigue identificando pensamiento con pensamiento lógico formal.

\footnotetext{
12 Ver Morin, Edgar. La cabeza bien puesta. Nueva Visión, Bs. As. 2002. pp. 92-93.

13 Asumimos que la Verdad existe, en la medida en que la realidad es una y es independiente de las personas $y$ sus posibilidades de conocer, esto hace posible a
}

En tanto dimensión profundamente humana, el pensamiento involucra la totalidad de la persona, con todas sus potencialidades y su historia personal, y pone en juego la libertad de cada ser humano: pensarse y pensar el mundo implica asumirse personalmente y como ser en el mundo, y asumir del mismo modo a los demás.

El pensamiento crítico y reflexivo posibilita conocer la realidad, comprenderla en sus estructuras y funcionamiento, y operar sobre ella racionalmente. Es un pensamiento que puede preguntarse por el sentido de las cosas y en particular del hombre, es el que puede ordenar acciones, ideas, etc. en función de valores que posibiliten al hombre ser más humano cada vez.

Un pensamiento verdaderamente reflexivo no puede soslayar la pregunta por el ser y por el sentido de la vida humana, y a su vez, la condición crítica del pensar no puede ser nunca entendida desde una lógica relativista $\mathrm{o}$ como un pensar instrumental: para ser verdaderamente crítico, el pensamiento debe preguntarse por lo específicamente humano. No, se trata de construir discursos, si bien la ciencia y la filosofía lo hacen, sino de la búsqueda de la Verdad ${ }^{13}$,

su vez, los esfuerzos de las ciencias y saberes por aproximar a los seres humanos a la verdad. En última instancia, si no hay Verdad que buscar, si no hay realidad que interpretar, la pretensión de conocimiento no tendría sentido. 
y esto necesariamente nos lleva a preguntarnos por el hombre, se trata entonces de un pensar que nos posibilite comprender al ser humano y a sus realizaciones en los contextos en que éstas se generan.

Este pensamiento, que definimos como crítico y reflexivo es un pensamiento filosóficoontológico y antropológico-, que se instala en el ámbito de lo profundamente humano, le posibilita a la persona preguntarse por sí misma y por los otros, y facilita la búsqueda del sentido de la propia vida.

La tarea de pensarnos a nosotros mismos es personal e intransferible, pero sin embargo, no es posible sin la mediación de otros. El lenguaje, que hace posible el pensamiento, sólo se aprende en la relación interpersonal; y las categorías a partir de las cuales nos pensamos a nosotros mismos son reinterpretadas a partir de un marco cultural determinado; y la cultura es producción humana, pero no sólo producción individual, sino social comunitaria -. La relación entre lenguaje y pensamiento no es meramente instrumental, sino que ambos se conforman uno al otro permanentemente, en un juego dialéctico: no es posible el lenguaje sin el pensamiento, pero tampoco habrá pensamiento sin lenguaje ${ }^{14}$.

\footnotetext{
${ }^{14}$ Si relacionamos con el planteo de Gardner acerca de las inteligencias múltiples, podemos ver como el autor identifica como característica de las inteligencias el uso del lenguaje verbal en algunos casos, preverbal en
}

El pensamiento crítico y reflexivo es un pensar situado, porque el ser humano es un ser en situación, es un ser histórico. Paulo Freire, alude a esta condición al referirse a la actitud con que el educador habrá de posibilitar la toma de conciencia de sus alumnos acerca de la historicidad del hombre, que constituye una de sus más ricas potencialidades:

Por ser el hombre un ser histórico, situado, con un aquí $y$ ahora, con un pasado determinado y con un futuro posible; la realidad humana, con sus modos de relación interpersonal, con sus construcciones teóricas y con sus productos; es siempre una realidad histórica. Y por ello mismo, es siempre contingente, provisoria, perfectible.

Hemos dejado para enunciar finalmente un rasgo esencial del pensamiento crítico y reflexivo tal como lo entendemos en este trabajo: se trata de un pensamiento liberador, que le permite a la persona emanciparse de miedos y oscuridades, le posibilita orientar su vida en una perspectiva teleológica, y así encontrarle sentido al esfuerzo cotidiano. El pensamiento crítico y reflexivo constituye al hombre como sujeto que decide su vida, que elige cómo y para qué vivirla. Esto requiere desnaturalizar las interpretaciones de sentido del

otros, y la creación de lenguajes formalizados en otras. Pero cada inteligencia remite a por lo menos un lenguaje. 
mundo y de la vida que nos vienen dadas por el entorno sociocultural. Conviene aclarar en este sentido que estas mediaciones socioculturales de sentido, limitan nuestra mirada y nuestra capacidad de pensar la realidad, pero a su vez la hacen posible. Esta condición paradojal es la que nos posibilita asumir la propia historia, personal y colectiva, y transformarla.

Un pensamiento crítico y reflexivo es aquel que contribuye a comprender la historicidad de lo humano, desnaturalizando la realidad y asumiendo que todo lo que ha construido el hombre y todo lo que ha asumido, es producto de decisiones más o menos libres. Permite, entonces, superar formas de pensamiento mágico - aquellas que remiten a fuerzas suprahistóricas o sobrenaturales - porque los procesos humanos se presentan como construcciones enraizadas en un tiempo y en un espacio determinados y como respuesta de un grupo humano a situaciones definidas; hace posible asumir críticamente - y no naturalmente la relación con los demás hombres, con el conocimiento y con el mundo.

\section{Enseñar historia y su aporte al pensamiento crítico y reflexivo}

El proceso de deconstrucción de la sociedad liberal-capitalista para los latinoamericanos - y la idea de desmontar la arqueología de lo obvio, de lo instituido, son tareas necesarias a la hora de encarar una mirada crítica y reflexiva sobre los hombres y su mundo: y en esta tarea, la Historia juega un rol insustituible en la medida en que nos permite tomar conciencia de la historicidad del mundo y por ello mismo de su contingencia. Entendiendo por tal no sólo la realidad física y material, sino el juego de relaciones interpersonales, el espacio de lo social y cultural que constituyen el ámbito del estar y posibilitan el ser del hombre.

La educación es el espacio privilegiado en el que se pone en juego de modo especial la dimensión relacional de los seres humanos. Este ser con otros que define al hombre y le posibilita humanizarse, encuentra un ámbito propicio en la institución educativa. El proceso educativo es siempre y en primera instancia un proceso relacional, y es además complejo, en él interactúan varias personas, se entrecruzan intencionalidades, aspiraciones, prejuicios y expectativas más o menos explícitas, según las circunstancias.

La educación, en tanto práctica que se orienta al hombre, y tiene como objetivo la formación integral de la persona, debe favorecer la formación del pensamiento crítico reflexivo. Así entra en juego el conocimiento histórico, como herramienta del proceso educativo; que posibilita el pensar situado - y por ello mismo desnaturalizado -, en la medida en que patentiza las realizaciones 
humanas como realizaciones históricas y por ello, contingentes; surgidas como respuesta a necesidades $^{15} \quad$ profundamente humanas, y permite la reflexión acerca del hacer del hombre; la aproximación a las intencionalidades, a las ideas y a los sentimientos que se ven implicados en las acciones de las personas.

Para lograr una educación que favorezca el pensamiento crítico reflexivo, es necesario otorgar espacio en la estructura curricular, al conocimiento histórico. Nos referimos no sólo al conocimiento de lo que ocurrió, conocimiento al que accedemos siempre mediado por la interpretación de alguien, o de algunos, que proporcionan ese relato, sino también a la posibilidad de desnaturalizar gestos, relatos, costumbres e ideas a las que adherimos como si naturalmente debieran ser así. Para que este modo de conocer sea posible, es necesario conocer no sólo "lo que ocurrió", sino las estrategias de construcción del relato histórico, ya que la Historia es en definitiva hermenéutica, debemos asumir que la interpretación es también acontecer histórico: se interpreta el pasado desde un aquí y un ahora cargado de intencionalidad.

\footnotetext{
15 No nos referimos solo a necesidades materiales, como se desprende de la lógica de este texto, sino que incluimos todo tipo de necesidades, entre ellas, especialmente la de encontrar el sentido de la propia vida y del mundo.
}

Admitir la historicidad del hombre y su producción no implica adoptar criterios relativistas, pero sí constatar la originalidad y la novedad que es propia de cada ser humano y que él imprime a sus realizaciones. Asumir la historicidad del hombre y el carácter contingente de todas las realizaciones humanas nos permite problematizar todas esas realizaciones sin aferrarnos a ninguna como ya dada.

Esto representa consecuencias importantes para la tarea educativa. Tarea de una enorme complejidad, y que no se agota en la transmisión de saberes o de prácticas culturales, sino en la posibilidad de aproximar a los alumnos a la búsqueda de sentido para la propia vida. Por ello, el proceso educativo, sólo es posible en tanto involucra a todo el hombre $^{16}$. Y constituye un proceso de humanización, que le permite a la persona conocerse profundamente a sí misma para poder poner sus capacidades al servicio de los demás. La educación tiene como finalidad posibilitarle al hombre su humanización para que pueda ser cada vez más plenamente él. No hay educación, entonces, que no se oriente a desarrollar aquellas condiciones y capacidades propiamente humanas. Podemos afirmar que toda educación debe atender al

\footnotetext{
16 "[...] estudiamos, aprendemos, enseñamos y conocemos con nuestro cuerpo entero. Con los sentimientos, con las emociones, con los deseos, con los miedos, con las dudas, con la pasión y también con la razón crítica. Jamás sólo con esta última." (Freire, 2002; p. 8).
} 
desarrollo del pensamiento crítico y reflexivo, ya que éste, condición propiamente humana, favorece el ejercicio pleno de la libertad.

\section{Algunas propuestas...}

Aprender a pensar y a pensarnos históricamente es un proceso. Y como tal, requiere de continuidad a lo largo de los años de la formación de jóvenes y de adolescentes, para posibilitar la maduración personal de actitudes y de ideas. Demanda además de la posibilidad real de interacción personal entre docente $y$ estudiantes, y también de los mismos estudiantes entre sí, y finalmente necesita de condiciones estructurales y organizativas que hagan posible respetar el tiempo personal de cada uno de los actores en el proceso de enseñanza y de aprendizaje.

Sin una cabal aproximación a la lógica y a los supuestos desde los que se construyen las disciplinas, resulta imposible para los alumnos adolescentes de la escuela secundaria, dialogar con las formulaciones que se les proponen, poner en discusión supuestos, dudar de afirmaciones que se sostienen como verdades acabadas. Sin esa aproximación se corre el riesgo de reemplazar una formación que se sustentó en la

\footnotetext{
17 Nos referimos al modelo de escuela positivista que se implementó en Argentina a partir de mediados del siglo XIX, que entendía por tal: el manejo de datos y de información, de conceptos sin intencionalidad valorativa, asumiendo el carácter descriptivo y acrítico del saber científico y disciplinar.
}

transmisión del conocimiento ${ }^{17}$; por una formación basada en la transmisión de modelos y construcciones ideológicas socialmente legitimadas, que bajo una pretensión de apertura a la reflexión crítica $^{18}$ genere la alienación de la persona.

Porque consideramos al hombre una persona, es que asumimos la riqueza y originalidad de cada ser humano, lo que nos obliga a admitir que existen diversas maneras de enseñar y de aprender, únicas para cada uno. Más allá de que podamos proponer criterios orientadores los procesos de enseñanza y de aprendizaje son personales, y las circunstancias históricas -sociales, culturales, económicas-, y personales afectivas, intelectuales, espirituales-, son componentes ineludibles de los mencionados procesos.

Es decir que sólo a título de sugerencias que orienten las decisiones de los profesores, enunciamos algunas líneas de acción para favorecer el pensamiento crítico y reflexivo;

- Potenciar la duda ante "verdades" que vienen dadas, la búsqueda de los argumentos que las sustentan, la ponderación de

\footnotetext{
${ }^{18}$ Insistimos en que no puede haber reflexión crítica sin el conocimiento de la lógica de las disciplinas y sin conciencia de los supuestos desde los cuales se construye el conocimiento científico o disciplinar.
} 
los mismos por parte de los estudiantes. La duda, constituye un paso metodológico, que hace posible el planteo de hipótesis interpretativas y explicativas, y que se sustenta en la búsqueda de la Verdad como aspiración de la persona humana.

- Trabajar con textos o documentos diversos, que les permitan observar que no hay una única manera de interpretar $y$ analizar un proceso o acontecimiento histórico, sino que siempre confluyen diferentes miradas, criterios, contextos personales, cosmovisiones que condicionan la interpretación. Esto, a su vez, permitirá desmitificar el valor del libro, muchas veces identificado por los adolescentes y jóvenes como portador de la verdad y no como una aproximación a ella.

- Propiciar la formulación de alternativas posibles para la explicación de un fenómeno. En este sentido se pueden discutir las alternativas con toda la clase, cotejar opiniones y enriquecer así

19 Resultan interesantes en este sentido experiencias relacionadas con lo lúdico, como pueden ser dramatizaciones, elaboración de formatos textuales no convencionales en la clase de Historia como pueden ser las historietas, afiches de propaganda política, entrevistas, etc.; sobre los que existen los puntos de vista personales.

- Potenciar, especialmente en los estudiantes del nivel secundario - y en particular de los cursos superiores -, el ejercicio de la reflexión y de la explicitación de los propios criterios para el análisis y la interpretación de los procesos históricos, como práctica habitual. Esto supone, desde luego, docentes entrenados en actitudes reflexivas hacia el conocimiento.

- Favorecer todas las actividades y experiencias creativas en la medida en que resulten pertinentes $y$ permitan la expresión de los adolescentes y jóvenes utilizando diversos lenguajes. Toda actividad que contribuye al desarrollo de la creatividad, facilita el pensamiento autónomo $\mathrm{y}$ divergente ${ }^{19}$.

Una propuesta formativa como esta, implica revisar y replantear la concepción acerca de los procesos de enseñanza y de aprendizaje, y aún más, el rol que juegan las ciencias y las disciplinas artísticas

propuestas didácticas, y de cuya eficacia en relación a generar una actitud crítica sobre el pasado, hemos sido testigos a través de experiencias de colegas y de los propios intentos. 
y deportivas, así como los saberes metacientíficos en la escuela. Es necesario redescubrir el valor de disciplinas y de prácticas pedagógicas que nuestro sistema educativo ha relegado, y que son imprescindibles en la medida en que se pretenda una formación integral de la persona humana.

Como historiadores y como docentes que enseñan Historia en la escuela secundaria, nos corresponde de modo especialísimo revisar el espacio y la tarea que le asignamos a la Historia en la formación de los estudiantes, de manera tal que nos permita repensar las condiciones de su enseñanza para atender efectivamente a una educación integral. Este proceso de revisión, es necesariamente gradual, $y$ presupone no sólo modificar las prácticas, sino también repensar categorías teóricas y revisar concepciones personales.

La pedagogía debe recuperar para que la educación sea verdaderamente integral, la dimensión humanizadora de la praxis educativa, que siempre es una práctica liberadora, y por esto mismo requiere de un pensamiento crítico y reflexivo que ponga al hombre en posesión de su vida, como protagonista lúcido y consciente de las decisiones con las que construye su historia personal y contribuye en la

\footnotetext{
20 A fines de 2017, el Congreso de la República de Colombia sancionó la ley 1874 que hace explícitos, entre las finalidades del estado colombiano en materia educativa la de:
}

construcción de la historia de la humanidad.

La enseñanza de la Historia, en el marco de la reflexión pedagógica que hemos propuesto, adquiere nuevas dimensiones: no se trata de proporcionar contenidos conceptuales acerca de los procesos históricos a los alumnos adolescentes de la escuela secundaria; sino de favorecer en ellos estrategias que les posibiliten el pensar históricamente, es decir, un pensar situado, desnaturalizando sistemas y estructuras ideológicas, sociales, económicas y políticas que se nos aparecen como "dados", como inherentes al hombre y a su realidad; para volver a situarlos como productos culturales -muy valiosos tal vez- de un tiempo y de una sociedad determinada. Como puede apreciarse la Historia tiene un potencial formativo realmente valioso, si es que nos decidimos a repensar también su lugar en la educación argentina y los criterios que sustentan su enseñanza.

La República de Colombia ha dado un paso decisivo en este sentido, al incorporar con rango de ley, la enseñanza de la Historia orientada a la formación de personas libres y responsables, críticas y reflexiva, comprometidas en transformar la sociedad en función de valores como la justicia, el reconocimiento y respeto por el otro $^{20}$. De esta manera una

“[...] restablecer la enseñanza obligatoria de la Historia de Colombia como una disciplina integrada en los lineamientos curriculares de 
propuesta teórica se convierte en política de estado. Ello implica un compromiso particular para los profesores: el de plasmar en las escuelas el texto de la ley.

\section{Referencias}

Bambozzi, E. (2005) Escritos pedagógicos. Ediciones del Copista, Córdoba.

Buber, M. (1994) Yo y tú. Ed. Nueva Visión, Bs As.

Congreso de la Nación Argentina. (1993). Ley Federal de Educación $\mathrm{N}^{\circ} 24195 / 93$.

Congreso de la Nación Argentina. (2006). Ley de Educación Nacional N² 26206/06.

Congreso de la República de Colombia. (2017). Ley 1874, del 27 de diciembre de 2017.

Freire, P. (2002) Cartas a quien pretende enseñar. $1^{\text {a }}$ ed. en portugués 1993. Bs. As, Siglo XXI.

Gardner, H. (1998). Inteligencias múltiples. La teoría en la práctica. Paidós, Bs. As.

Guardini, R. (1967). Mundo y Persona. Ensayos para una teoría cristiana del hombre. $1^{\mathrm{a}}$ ed

las ciencias sociales en la educación básica y media, con los siguientes objetivos:

a) Contribuir a la formación de una identidad nacional que reconozca la diversidad étnica cultural de la Nación colombiana.

b) Desarrollar el pensamiento crítico a través de la comprensión de los procesos históricos y en castellano 1963. Ed. Guadarrama, Madrid.

Habermas, J. (1990). La lógica de las ciencias sociales. Tecnos, Madrid.

Morin, E. (2002). La cabeza bien puesta. Nueva Visión, Bs. As. sociales de nuestro país, en el contexto americano y mundial.

c) Promover la formación de una memoria histórica que contribuya a la reconciliación y la paz en nuestro país". 


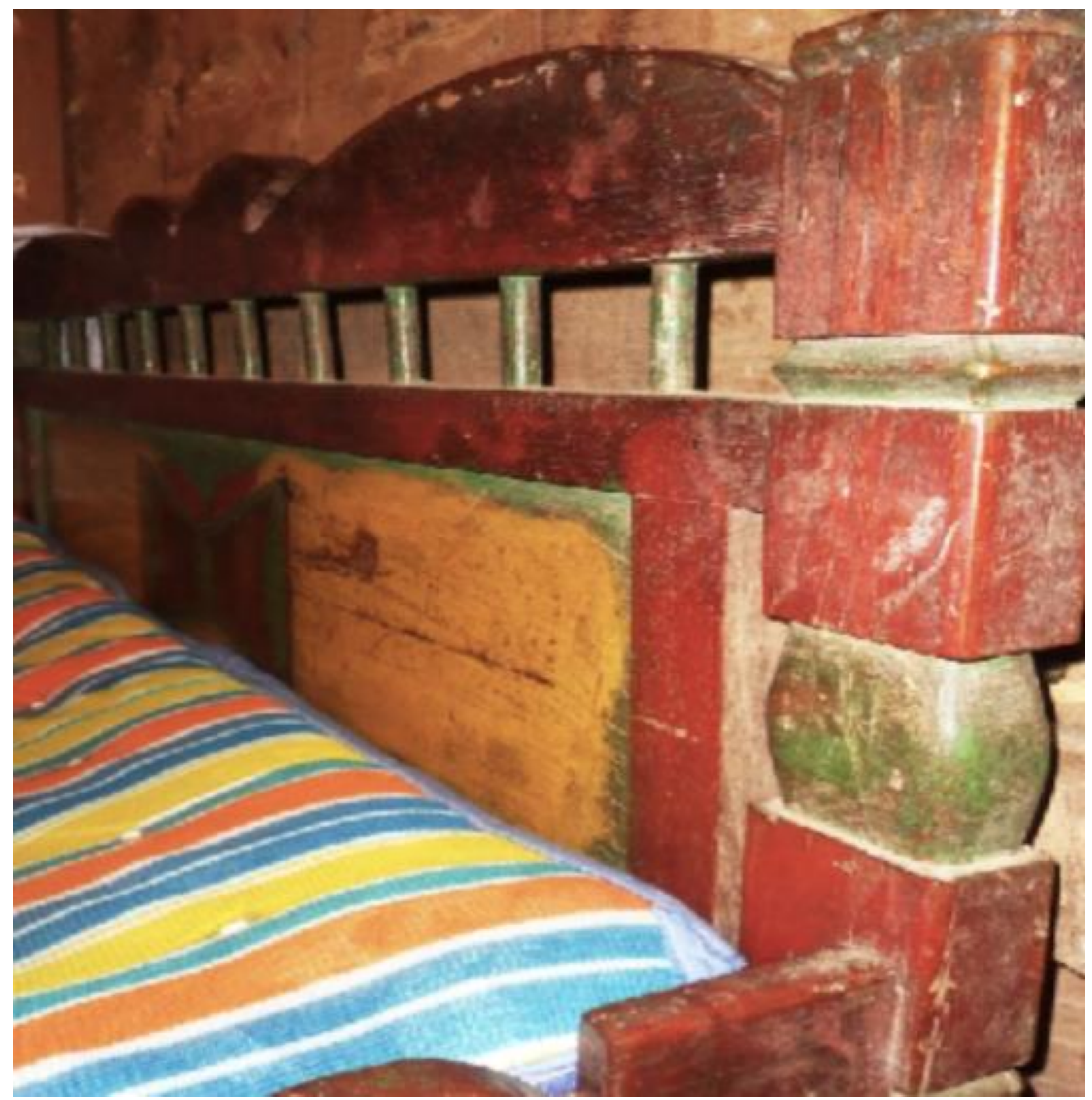

Número de la obra: 9

Título: "sobre camas"

Autor: Yenny Banesa Bonilla

Lugar: Zona rural municipio de Baraya

Finca: Chapetón

Fecha de captura: Febrero 28 de 2014

Técnica: Fotografía digital

Fuente: Bonilla, Y. B. (2014). Sobre camas. Una mirada estética popular del municipio de Baraya. Tesis de pregrado. Universidad Surcolombiana. Neiva, 2014. p.78. 